\title{
An experimental study of transitional pulsatile pipe flow
}

\author{
R. Trip, ${ }^{\text {a) }}$ D. J. Kuik, J. Westerweel, and C. Poelma ${ }^{\text {b) }}$ \\ Laboratory for Aero and Hydrodynamics (3ME-P\&E), Delft University of Technology, \\ Leeghwaterstraat 21,2628 CA Delft, The Netherlands
}

(Received 2 June 2011; accepted 2 December 2011; published online 6 January 2012)

\begin{abstract}
The transitional regime of a sinusoidal pulsatile flow in a straight, rigid pipe is investigated using particle image velocimetry. The main aim is to investigate how the critical Reynolds number is affected by different pulsatile conditions, expressed as the Womersley number and the oscillatory Reynolds number. The transition occurs in the region of $\mathrm{Re}=2250-3000$ and is characterized by an increasing number of isolated turbulence structures. Based on velocity fields and flow visualizations, these structures can be identified as puffs, similar to those observed in steady flow transition. Measurements at different Womersley numbers yield similar transition behavior, indicating that pulsatile effects do not play a role in the regime that is investigated. Variations of the oscillatory Reynolds number also appear to have little effect, so that the transition here seems to be determined only by the mean Reynolds number. For larger mean Reynolds numbers, a second regime is observed: here, the flow remains turbulent throughout the cycle. The turbulence intensity varies during the cycle, but has a phase shift with respect to the mean flow component. This is caused by a growth of kinetic energy during the decelerating part and a decay during the accelerating part of the cycle. Flow visualization experiments reveal that the flow develops localized turbulence at several random axial positions. The structures quickly grow to fill the entire pipe in the decelerating phase and (partially) decay during the accelerating phase. (C) 2012 American Institute of Physics. [doi:10.1063/1.3673611]
\end{abstract}

\section{INTRODUCTION}

The transition of pulsatile flow from the laminar to the turbulent regime has drawn the attention of many investigators. One of the prime motivations for these efforts stems from the question whether flow in the cardiovascular system, in particular in the large arteries, can become turbulent (either in normal conditions or due to exercise or diseases). Turbulent flow-or more generally "disturbed flow"- and the resulting fluctuating wall shear stresses have been associated with the etiology of a range of cardiovascular diseases. ${ }^{1}$ Conditions in the large arteries are such that a corresponding mean flow would be in the transitional regime-during parts of the cycle, the instantaneous Reynolds number significantly exceeds the values that are commonly associated with transition. Rather than dealing with the full, complex biomechanical geometry, we will simplify the problem to a study of the transition of a pulsatile flow in a straight, rigid pipe. Effects due to, for instance, vessel curvature, bifurcations, wall distention, wall roughness, and non-Newtonian behavior of blood are thus ignored. This isolates the core fundamental fluid mechanical problem, which is closely linked to the research of transition of steady pipe flow. As the mechanisms of transition of steady flow is still under debate, ${ }^{2-4}$ this paper will focus mainly on the question when a pulsatile flow becomes turbulent, though this is of course closely related to the underlying mechanisms.

A large number of studies have been carried out to clarify the fluid mechanical aspects of pulsatile flow. The basic concepts and an extensive review are described by Çarpinlioğlu and Gündoğdu. ${ }^{5}$ Laminar pulsatile flow is well understood and an analytical solution exists. ${ }^{6,7}$ For purely

\footnotetext{
${ }^{a)}$ Present address: Linné Flow Centre, KTH Mechanics, Stockholm, Sweden.

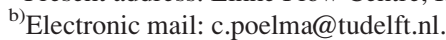


oscillating flow (i.e., no mean flow component), transition can be predicted with reasonable accuracy by evaluating the product of the Reynolds number and a frequency parameter ${ }^{5,8}$ (the latter usually referred to as Womersley number, see Sec. II). This product is then the critical Reynolds number, at which the flow becomes unstable. Once a mean flow component is added, the body of experimental evidence is unfortunately not without contradictions and the mechanisms and the relevant parameters are poorly understood. These contradictions motivate ongoing interest in pulsatile flow ${ }^{9}$ and highlight the demand for more experimental data. ${ }^{5,10,11}$

The earliest studies, which relied on pressure drop measurements, showed that the mean Reynolds number for which transition occurs decreased from $R e=2200-2400$ (for steady flow) to as low as 1500 when the pulsatile component is increased. ${ }^{12}$ In vivo studies in dog aortas using hot film anemometry confirmed this behavior: both Nerem and Seed ${ }^{13}$ and Falsetti et al. ${ }^{14}$ concluded that the critical Reynolds number decreases with increasing Womersley number $(\alpha)$. The latter study suggested that the ratio of mean Reynolds number and Womersley number can be used as a criterion, with $R e / \alpha>200$ indicating turbulent flow. This result is closely related to the criteria defined for purely oscillating flows. However, Stettler and Hussain ${ }^{15}$ found that only for relatively low values of $\alpha(<3)$ transition occurs at lower Reynolds number. For the range $(4<\alpha<10)$, transition is actually delayed to a higher Reynolds number. Based on a series of in vitro measurement that mimic the physiological conditions, Peacock et al. ${ }^{16}$ found that the critical Reynolds number correlates with a non-dimensional amplitude parameter and the Womersley number as a power law.

A critical Reynolds number suggests transition is an instantaneous event. However, for both steady ${ }^{17}$ and unsteady flow ${ }^{18}$ transition occurs over a range of mean Reynolds numbers, in which an increasing number of isolated turbulent structures is formed. This intermittency of turbulence was found to be a function of the mean velocity, the frequency, and the amplitude of the pulsatile flow. ${ }^{18,19}$

Stettler and Hussain ${ }^{15}$ describe another regime for which pulsatile flow is of intermittent nature: for certain values of the Womersley number, modulation amplitude and mean velocity, the turbulent structures do no longer occur randomly, but are phase-locked. The occurrence of phasedependent turbulence is often claimed to be due to the stabilizing effect of accelerating flow, whereas the decelerating flow leads to transition more readily. Ramaprian and $\mathrm{Tu}^{20}$ found that the turbulence intensity can experience large phase shifts for different frequencies, whereas Iguchi et $a l^{21}$ suggests that there is no phase difference between the turbulence intensity and the mean velocity. Furthermore, Eckmann and Grotberg ${ }^{22}$ found that turbulence can occur in confined regions near the walls, while the core remains laminar.

\section{A. Aim and scope of this study}

As will be clear from aforementioned brief survey, it remains inconclusive if and how the transition from laminar to turbulent flow (based on the mean Reynolds number) is affected by pulsatile flow conditions - especially in the physiologically relevant regime. The goal of this study is to measure the transitional regime for different frequencies of a sinusoidal periodic signal with a non-zero mean within a rigid straight cylindrical geometry. The use of a single sinusoidal velocity component is again a simplification of the flow in the cardiovascular system, but it gives insight in the fundamentals of the transition without having to deal with the added parameter space of a complex pulse shape.

A pulsating flow at relatively low Reynolds number will be generated in a range of parameters (see Sec. II). As the flow is linearly stable, ${ }^{23,24}$ a controlled perturbation is generated by placing a ring-type constriction at the entrance of the flow. This can also be interpreted as e.g., the presence of (artificial) heart valves at the start of the aorta. ${ }^{25}$ The turbulence intensity will be used as a metric for the presence of turbulence. We use this parameter to determine if the flow is turbulent throughout the entire cycle or only during a particular phase of the cycle.

A complicating factor in the comparison and unification of previous literature results is the fact that they are nearly always obtained using single-point techniques (e.g., laser Doppler anemometry or hot-wire anemometry). As will become clear in this study, this will make it difficult to distinguish between (local) mean and fluctuating velocity components. This complicates the 
estimation of e.g., the turbulent intensity to determine whether the flow is laminar or turbulent. In this study, we use particle image velocimetry. This, as will become clear in Sec. III, avoids the problems due to the intermittency of the flow.

The results are presented in two main sections: in Sec. IV, the transitional regime is described. In this regime, the flow contains an increasing number of isolated turbulent structures ("puffs") for an increasing mean Reynolds number, before finally becoming fully turbulent. In Sec. V, we present results for the characteristics of such a turbulent flow, in particular its behavior during the pulsating cycle. Flow visualization experiments will be carried out to further support the conclusions drawn upon the turbulence intensity results. Apart from providing a method of judging whether the flow is turbulent or not, the particle image velocimetry (PIV) technique also allows instantaneous measurement of e.g., radial profiles of turbulent kinetic energy and Reynolds transport terms.

\section{THEORETICAL BACKGROUND}

From dimensional analysis, it follows that three dimensionless parameters are needed to describe a sinusoidal pulsatile flow. ${ }^{16}$ The parameters chosen here are the mean Reynolds number $R e_{m} \equiv 2 u_{s} R / \nu$, the oscillatory Reynolds number $R e_{o} \equiv 2 u_{o} R / \nu$, and the Womersley number $\alpha \equiv R \sqrt{(2 \pi f / \nu)}$. In these definitions, $R$ is the radius of the pipe and $\nu$ the kinematic viscosity. The axial velocity $u$, composed of a steady velocity component $u_{s}$ and an oscillatory velocity component $u_{o}$, and the frequency $f$ are defined by Figure 1 . The Reynolds numbers describe the ratio of inertial to viscous forces, while the Womersley number gives the ratio of transient inertial force to viscous forces. ${ }^{26}$ Other dimensionless groups could be chosen, of which the ratio $R e_{o} / R e_{m}$ is also commonly used. This parameter describes the ratio of oscillating and mean velocity component. Where relevant, we will also report the value of this dimensionless number. This study will show that there is no particular set of parameters that has a clear advantage.

For an axisymmetric flow of an isotropic, incompressible, Newtonian fluid without external forces an analytical solution of the Navier-Stokes equations can be derived for fully developed laminar pulsatile pipe flow; this is often referred to as Womersley flow. The axial velocity $u$ as function of radial position $r$ and time $t$ is given by ${ }^{7}$

$$
u(r, t)=\frac{p_{s}}{4 \mu}\left(r^{2}-R^{2}\right)+\frac{p_{o}}{i \mu} \frac{R^{2}}{\alpha^{2}}\left\{1-\frac{J_{0}\left(\alpha \frac{r}{R} i^{\frac{3}{2}}\right)}{J_{0}\left(\alpha i^{\frac{3}{2}}\right)}\right\} e^{i \omega t} .
$$

In this equation, $p_{s}$ is the time-independent pressure gradient, $p_{o} \exp (i \omega t)$ the time-dependent pressure gradient, and $\mu$ the dynamic viscosity. In the first term, the classic parabolic Poiseuille flow can be recognized, while the second term represents a flatter, phase-lagged profile representing the effects of transient inertia (note that $J_{0}$ is a Bessel function of the first kind of zeroth order).

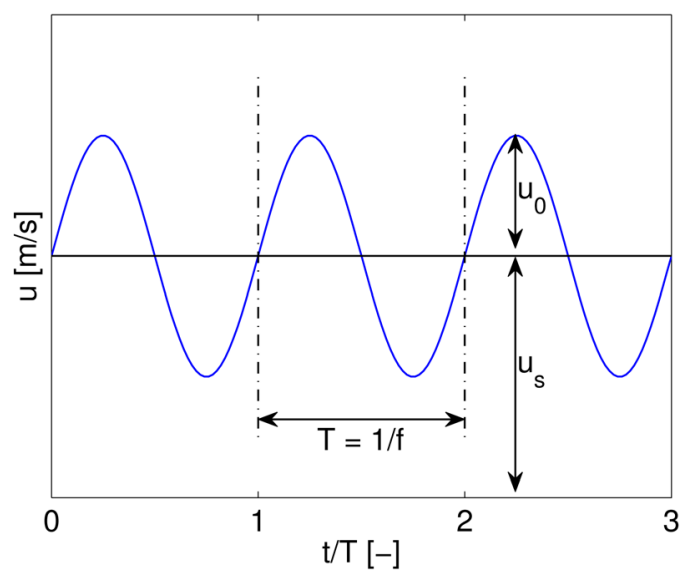

FIG. 1. (Color online) The sinusoidal axial velocity $u$ and the definition of the mean velocity $u_{s}$, the oscillatory velocity $u_{o}$, the frequency $f$, and corresponding period $T$. 
To interpret the results of the measurements of a (potentially turbulent) pulsatile flow, we make use of the decomposition of the flow parameters into contributions from the mean, periodic and non-periodic components, and a measurement error. ${ }^{27}$ Ignoring terms that are zero by definition, we find the following for the axial $(u)$, radial $(v)$, and azimuthal $(w)$ velocity components:

$$
\begin{gathered}
u(r, t)=u_{s}(r)+u_{o}(r, t)+u^{\prime}+\Delta u(t), \\
v(t)=v^{\prime}+\Delta v(t), \\
w(t)=w^{\prime}+\Delta w(t) .
\end{gathered}
$$

The subscripts $s$ and $o$ refer to the (long) time mean velocity ("steady") and the periodic ("oscillating") component, respectively. These will be introduced more formally in Sec. III. In Eqs. (2)-(4), $u^{\prime}, v^{\prime}$, and $w^{\prime}$ are the turbulent fluctuations of the velocity. These non-periodic components are a function the axial, radial, and azimuthal position $(x, r, \theta)$; this has been omitted here for brevity. The same holds for the final components, $\Delta u(t), \Delta v(t)$, and $\Delta w(t)$, which are caused by measurement errors and variations in the flow due to e.g., pump fluctuations.

The bulk velocity $u_{b}$ of the laminar case is given by

$$
u_{b}(t)=\frac{1}{\pi R^{2}} \int\left[u_{S}(r)+u_{o}(r, t)\right] 2 \pi r d r .
$$

The final parameter that is introduced is the turbulence intensity, a common parameter to characterize turbulence, which is defined as

$$
I=\frac{1}{u} \sqrt{\frac{1}{3}\left(\left\langle u^{\prime 2}\right\rangle+\left\langle v^{\prime 2}\right\rangle+\left\langle w^{\prime 2}\right\rangle\right)},
$$

with $u$ representing a reference velocity value. The reference velocity used is either the bulk velocity or the centerline velocity, which are both a function of time. The axial and radial components of the turbulence intensity are defined by $I_{a}=\sqrt{\left\langle u^{\prime 2}\right\rangle} / u$ and $I_{r}=\sqrt{\left\langle v^{\prime 2}\right\rangle} / u$, respectively. $I_{a}$ and $I_{r}$ will be used later on to show the effects of intermittency.

\section{EXPERIMENTAL TECHNIQUES}

\section{A. Flow facility}

A schematic illustration of the experimental facility is shown in Figure 2. A pulsatile flow is generated by a gear pump (Liquiflo Rotogear Sealed Pump 37 F). The volumetric flow rate is monitored by a mass flow meter (Krohne Optimass MFM 7050 K T15). A settling chamber, consisting of a combination of screens and a honeycomb, is used to remove pump-induced swirls. ${ }^{28}$ The outlet of the settling chamber consists of a contraction to ensure a smooth inflow into a smooth rigid perspex pipe with an inner diameter of $d=40 \mathrm{~mm}$. The length of the pipe $\left(L_{p}\right)$ is 6 $\mathrm{m}$, equivalent to 150 diameters. The entrance length for a laminar oscillating pipe flow can be estimated as $L / D=0.049 R e_{m}{ }^{5}$ which suggests that the flow can be considered to be fully developed at the measurement location until $R e_{m}=3100$. Some experiments will be performed at a higher value of $R e_{m}$, but these are in the turbulent regime; this makes the entrance length requirements less stringent than the laminar case.

The orifice plate, which facilitates transition to turbulence, has a diameter of $d_{o r}=30 \mathrm{~mm}$, i.e., an area reduction of $44 \%$, and is placed immediately behind the smooth entrance. The size of the ring-type constriction is based on an a previous study by Durst and Ünsal ${ }^{29}$ who provide an indication of the mean Reynolds number at which transition is expected to occur. We should note here that the type of disturbance may have an influence on the critical Reynolds number. However, the main focus of this study is to investigate the role of pulsatile flow on transition (while keeping the disturbance mechanism constant). Other disturbance mechanisms may shift the transition behavior to somewhat higher or lower Reynolds numbers, but it is expected that the influence of 


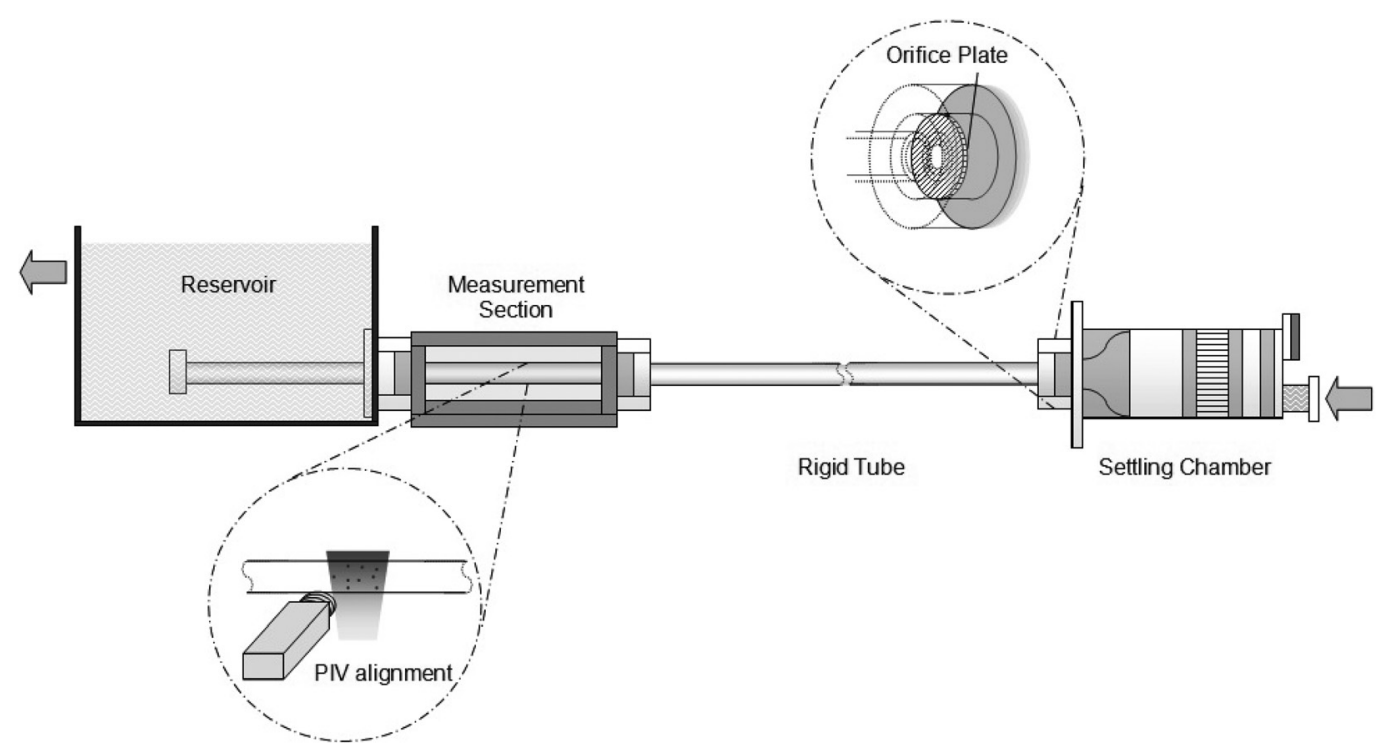

FIG. 2. A schematic representation of the experimental facility.

the pulsatile flow parameters remains unaffected. The measurement section is at the far downstream side of the pipe. Here, the pipe is enclosed by a rectangular glass box filled with water to reduce the optical distortion due to the curved pipe walls. The rigid pipe within the measurement section is replaced by a thin-walled glass section with the same inner diameter to further optimize optical access. The temperature of the working fluid (water) is somewhat influenced by temperature fluctuations of the surrounding air, even though most sections are insulated. Therefore, the viscosity of water is corrected a posteriori using the actual water temperature during each experiment.

\section{B. Measurement technique}

Several techniques can be used to measure the velocity characteristics of a pulsatile flow. An overview of the advantages and limitations of these techniques is given by Nabavi and Siddiqui. ${ }^{11}$ Planar (2D) PIV (Ref. 30) is chosen here because PIV provides quantitative evaluation of twodimensional velocity fields. In order to use PIV in a pulsatile flow, the image recording has to be synchronized with the oscillating flow to capture velocity field at a desired phase. Therefore, the camera and the laser are triggered phase-locked with the pump by a SRS Digital Delay and Pulse Generator DG535.

Tracer particles are required to visualize the flow of the optical transparent working fluid. Here, 10-12 $\mu \mathrm{m}$ hollow glass particles are used (Sphericell 110P8, Potters Industries). A dual cavity Nd:Yag laser system (New Wave Solo PIV) is used for illumination. The laser sheet with a thickness of $0.5 \mathrm{~mm}$ is aligned parallel to the axis of the tube and illuminates an area of $60 \mathrm{~mm}(L) \times 50 \mathrm{~mm}(H)$. A 12-bit CCD camera (LaVision FlowMaster $3 \mathrm{~S}$ ) with $1280 \times 1024$ pixels is used. The camera is oriented perpendicular to the light sheet to record both the axial (stream-wise) and radial velocity components.

The PIV gray-value images are processed using an in-house code. This code is a multipass PIV FFT-correlation based algorithm as described by Westerweel et al. ${ }^{31}$ Outliers are detected by a normalized median test ${ }^{32}$ and replaced by a linear interpolation algorithm. The initial interrogation size is $128 \times 64$ pixels which is reduced to a final window size of $32 \times 16$ pixels during four iterations in order to improve the window pre-shift and thus the signal-to-noise ratio. The measurement volume for each velocity vector then becomes $V=0.5 \times 1 \times 0.7 \mathrm{~mm}^{3}$ at these parameters. No correlation averaging is used because of the expected turbulent nature of the flow. In the radial direction, a 50\% window overlap is used to improve the resolution in this direction. In the axial direction, overlap is not used to avoid biasing the turbulence statistics. ${ }^{33}$ 


\section{Data processing}

As shown in Eqs. (2)-(4), the velocity at each instance can be decomposed in a number of contributions. PIV can be used to distinguish each of these contributions. We formally introduce the definitions here to avoid confusion when dealing with the different averages that will be used. Measurements are conducted for a number of periods of the sinusoidal flow, from now on referred to as "cycles." A total of $N$ cycles is recorded, where $n$ indicates the number of the cycle. Within each of these cycles, we record $M$ velocity fields, so we obtain a total number of $N \times M$ fields. The number of cycles is $N=50$, with the number of velocity fields $M=8$, unless stated otherwise.

Each of the fields as an unique index $i$ (ranging from 1 to $N \times M$ ). Using this notation, we represent a single vector field as $V_{i}(x, r)$; this vector field contains the axial and radial components $u_{i}(x, r)$ and $v_{i}(x, r)$, with $x$ and $r$ the axial and radial position. We define the phase-averaged velocity field (at phase index $m$ ) as

$$
\Psi_{m}(x, r)=\frac{1}{N} \sum_{n=0}^{N-1} V_{M n+m}(x, r) .
$$

Due to the assumption of fully developed flow, Eq. (1) is the solution to a linear governing equation; this also means that the steady and unsteady parts of pulsatile flow are independent in case of laminar flow. The steady part $(\bar{\Psi})$ can be computed by averaging all realizations, which must also be equal to the average of each of the phase-averaged velocity fields

$$
\bar{\Psi}(x, r)=\frac{1}{N \times M} \sum_{i=1}^{N \times M} V_{i}(x, r)=\frac{1}{M} \sum_{m=1}^{M} \Psi_{m}(x, r),
$$

from which the phase-averaged unsteady (oscillating) part follows immediately:

$$
\langle\Psi\rangle_{m}(x, r)=\Psi_{m}(x, r)-\bar{\Psi}(x, r) .
$$

This decomposition of the instantaneous velocity fields $V_{i}$ into the averaged velocity fields $\bar{\Psi}$ and $\langle\Psi\rangle$ is a first step to obtain the flow characteristics. The steady velocity and oscillatory velocity component are expected to be constant in the stream-wise $(x)$ direction. Their average can be computed by integrating all elements at a particular radial location $(r)$ for each phase index $(m)$

$$
\begin{aligned}
\bar{\Psi}(r) & =\frac{1}{L} \int \bar{\Psi}(x, r) d x, \\
\langle\Psi\rangle_{m}(r) & =\frac{1}{L} \int\langle\Psi\rangle_{m}(x, r) d x,
\end{aligned}
$$

with $L$ the width of the field of view. These integrals are evaluated as summation due to the discretized nature of the velocity measurements.

Finally, the velocity fluctuation field $\Psi_{i}^{\prime}(x, r)$ for an instantaneous velocity field $\Psi_{i}(x, r)$ can be found by subtracting the "long-time" mean $\Psi$ and average oscillating components $\langle\Psi\rangle_{m}$

$$
\Psi_{i}^{\prime}(x, r)=V_{i}(x, r)-\langle\Psi\rangle_{m}(x, r)-\bar{\Psi}(x, r) .
$$

The velocity fluctuations $\left(u^{\prime}, v^{\prime}\right.$; the components of the velocity field $\left.\Psi_{i}^{\prime}\right)$ are usually characterized using the root-mean-square (RMS) velocity, which is equal to the standard deviation of the cycle-to-cycle variation. However, besides velocity fluctuations, the RMS velocity includes measurement noise $(\Delta u, \Delta v)$ as well (as shown in Eqs. (2) and (3)). Because PIV provides spatial velocity information of a correlated signal (e.g., a turbulent flow), uncorrelated noise can be corrected for by evaluating the correlation function of the signal. ${ }^{33}$ The uncorrelated noise contributes to a delta peak at the origin only in the autocovariance function $R$. For the axial component, the discrete form of this function is defined as

$$
R_{x x}(s, r)=\frac{1}{2 k-1} \sum_{s=-k+1}^{k-1} u^{\prime}(x, r) u^{\prime}(x+s, r) .
$$


In this definition, $k$ is the number of velocity vectors in the axial direction. In a turbulent flow, the shape of $R_{x x}$ for small separations is known to be a parabola, with its maximum at $s=0 .{ }^{34}$ Therefore, a correction can be obtained by extrapolation of the parabola through a few adjacent points near the center; three points are used for the present study. The maximum value of the parabolic fit (the value at $s=0$ ) is equal to the variance $\left\langle u^{\prime 2}\right\rangle$ of the signal. Typically, the noise contribution was $8 \%$ of the signal. As the relative amount of measurement noise is influenced by the flow field, this noise correction is essential to avoid a biased view of the flow statistics. Similar expressions as derived here for the axial component can also be defined for the radial velocity component $v^{\prime}$.

\section{TRANSITION OF PULSATILE FLOW}

\section{A. Laminar flow}

Measurements are conducted for both steady and unsteady flow within the range of mean Reynolds number $R e_{m}$ for which transition is expected $\left(R e_{m}=2000-3500\right)$. The number of images $N$ recorded is fifty, because the velocity data converged sufficiently for this number in preliminary experiments, as shown in Figure 3.

Before looking at the turbulence intensity, it is worthwhile to compute the velocity profiles of laminar steady, oscillatory, and pulsatile flow and compare these results with the theoretical shape given by Eq. (1). Examples of instantaneous velocity profiles of typical pulsatile flow are shown in Figure 4. In this figure, the mean component $(\times)$, the oscillating component $(\cdot)$, and the sum ( $\bigcirc$, "pulsatile") are shown for both a laminar and a turbulent case. The data has been normalized with the centerline velocity for each case. For the laminar case, the continuous lines indicate the theoretical prediction given in Eq. (1). Only the phase of the theoretical predictions has been adjusted to match the experimental data, as this particular set of data was not phase-locked. For the turbulent, the velocity distribution is more uniform as a result of more efficient momentum transport due to eddies.

The laminar velocity profile is skewed somewhat, most likely due to non-ideal inlet conditions that persist in the laminar case for high Reynolds numbers. However, this very minor skewness is not expected to influence the results of this study.

\section{B. Transition of steady flow}

The transition of steady flow is examined first. The turbulence intensity is measured for a mean Reynolds number from $R e_{m}=2000$ to $R e_{m}=3500$. Because the volumetric flow rate is controlled, the mean Reynolds number is corrected for the temperature dependence of the kinematic viscosity. The turbulence intensity is the statistical average over fifty realizations.

In Figure 5, the turbulence intensity at the centerline based on the, respectively, the radial and axial velocity fluctuations are shown. Error bars denote the standard error of the mean. For the axial component, a large overshoot is shown for $R e_{m}=2388$, a phenomenon reported before. ${ }^{29}$

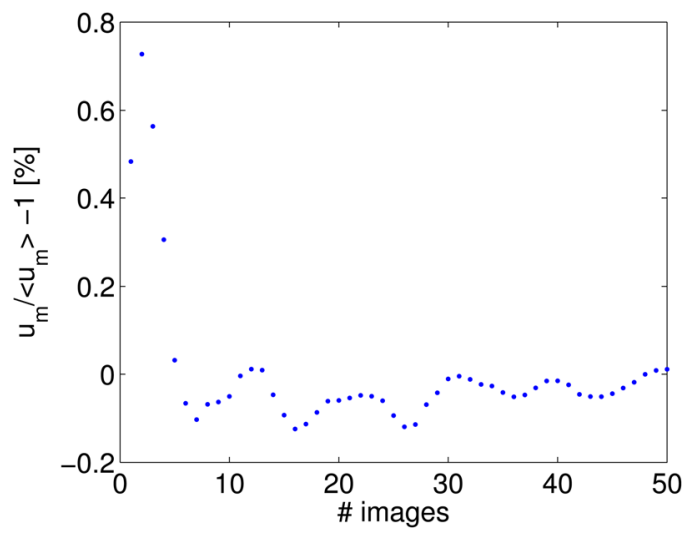

FIG. 3. (Color online) The mean velocity $u_{m}$ as function of the number of images used to average. The data are normalized using the value obtained using all available images. 


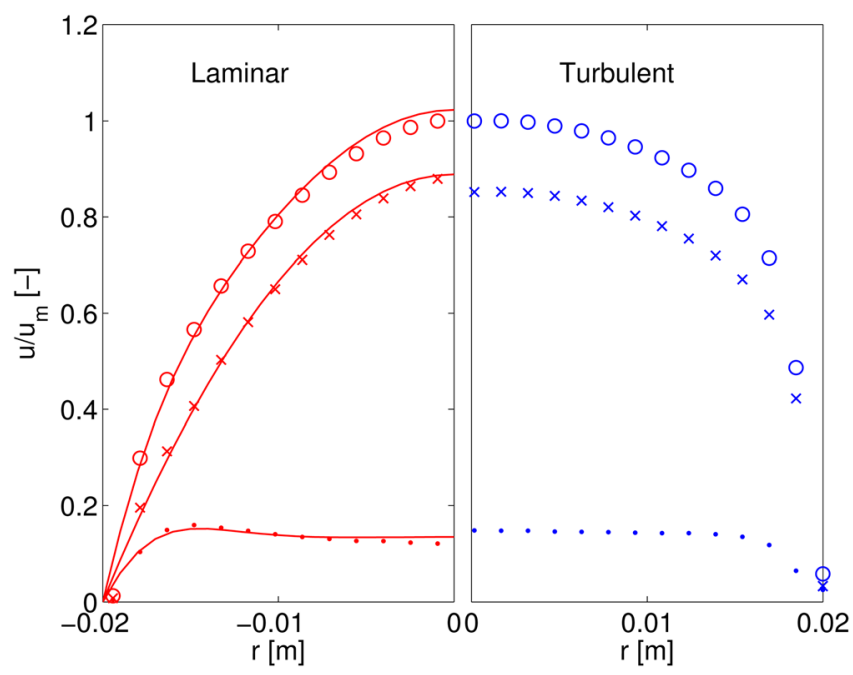

FIG. 4. (Color online) Left: Measured and theoretical steady $u_{s}(\times)$, oscillatory $u_{o}(\cdot)$, and pulsatile $u_{m}(\bigcirc)$ velocity profiles of a laminar pulsatile flow $\left(\alpha=10, R e_{m}=2160\right.$, and $\left.R e_{o}=610\right)$. Right: Measured steady $u_{s}(\times)$, oscillatory $u_{o}(\cdot)$, and pulsatile $u_{m}(\bigcirc)$ velocity profiles of a turbulent pulsatile flow $\left(\alpha=10, R e_{m}=3160\right.$, and $\left.R e_{o}=610\right)$.

Because of the more efficient momentum transfer, the mean velocity component of turbulent flows is smaller compared to laminar flow. Due to the intermittent nature within the transitional regime, the ensemble average of the mean velocity component is biased and the result for the velocity fluctuations increases as a result (see also Figure 6 for a schematic illustration). Although the overshoot might be considered physical because the turbulence intensity is a statistical quantity, the overshoot does not give a clear value for the intermittency. One way to avoid the overshoot is by looking at the radial velocity component, for which the mean component is equal to zero for both the laminar and turbulent flow state. As a result, no overshoot is shown for radial component of the turbulent intensity as shown in Figure 5. To get a better understanding of turbulence during transitional flow, the turbulence intensity based solely on the radial component $v^{\prime}$ is more appropriate here and will be used for the remainder of this section. In Sec. IV C, and in particular in Figure 6, an alternative method to overcome the overshoot will be presented, based on the subtraction of the line average in the axial direction of a PIV recording.

The turbulence intensity normalized by the centerline velocity is $I_{r} \approx 1 \%$ for a mean Reynolds number up to $R e_{m} \approx 2400$. The turbulence intensity of this flow, which is still considered to be laminar, is a result of all contributions not related to turbulence like measurement errors and small fluctuations in the pump output. An increase in intensity can be observed in a range that is referred

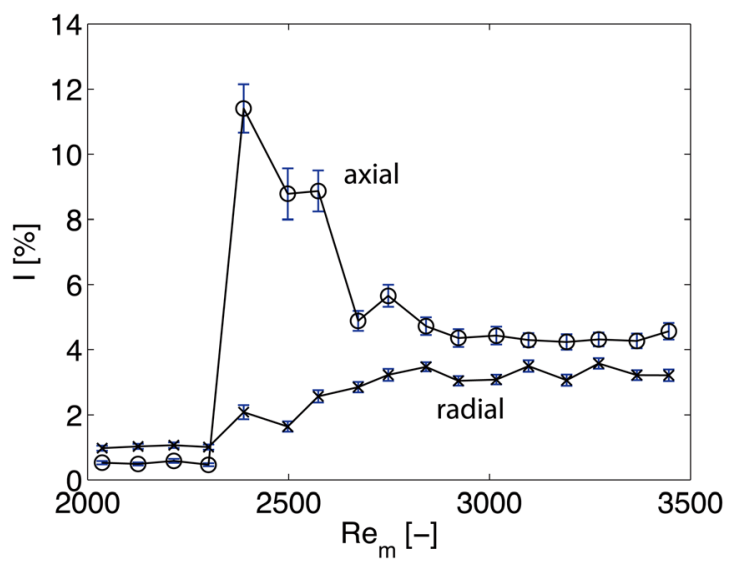

FIG. 5. (Color online) Turbulence intensity $I$ at the centerline, determined by normalizing the velocity fluctuations with the centerline velocity, as function of the mean Reynolds number $R_{m}$ for steady flow. The axial $I_{a}(\bigcirc)$ and radial $I_{r}(\times)$ component of the turbulence intensity are shown separately. 


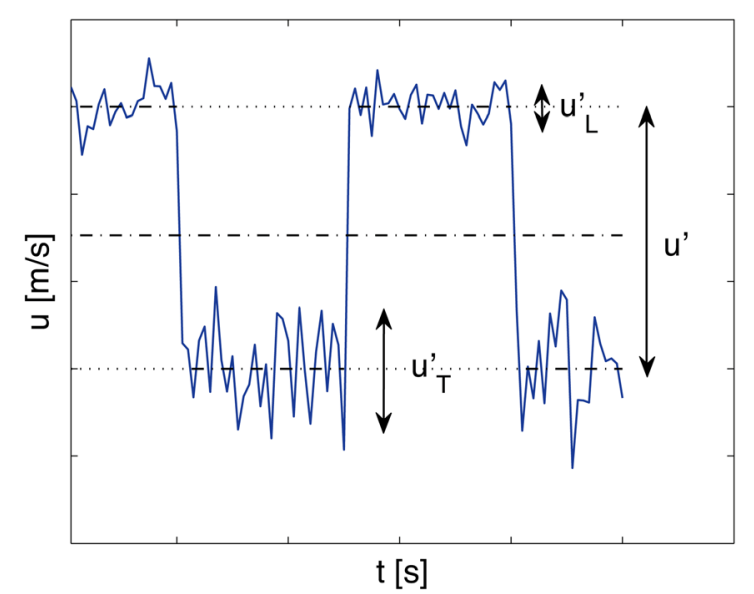

FIG. 6. (Color online) Schematic illustration of the effect of intermittency on the resultant turbulence intensity. The presence of both laminar and turbulent flow in a measurement results in a biased ensemble average and velocity fluctuation $u^{\prime}$. Subtracting a line average in the axial direction will give unbiased laminar $u_{L}^{\prime}$ and turbulent $u_{T}^{\prime}$ velocity fluctuations only.

to as transitional $\left(R e_{m} \approx 2400-2800\right)$. Within this region, puffs occur randomly in time. Evidence for the presence of these puffs will be presented in Sec. IV C. For now, it suffices to mention for an increasing $R e_{m}$; an increasing number of puffs occurs until the flow has become turbulent at every instance which is the case for $R e_{m}>2800$. The turbulence intensity at this Reynolds number normalized by the centerline velocity is $I_{r} \approx 3 \%$. This range is consistent with the range expected based on the flow conditions and the orifice plate used. ${ }^{29}$ The boundaries for laminar, transitional, and turbulent flow measured here will be used as a reference for the results of unsteady flow.

\section{Transition of unsteady flow}

A straightforward calculation of the turbulence intensity is not a satisfactory metric due to the intermittent character of the flow in the transition range. A solution is to subtract the line average in the axial direction for each fluctuating velocity field

$$
\Psi_{m o d, i}^{\prime}(x, r)=\Psi_{i}^{\prime}(x, r)-\frac{1}{L} \int \Psi_{i}^{\prime}(x, r) d x
$$

One should notice this is only valid if the turbulent structures are large compared to the width of the field of view. A typical puff is over 20 diameters in length and, therefore, extends over multiple measurement volumes. ${ }^{17}$ The error made because only part of the puff is within the field of view is small for that reason. The main motivation for subtracting the line average is that the velocity fluctuation is now corrected for the biased ensemble average in case of intermittent turbulence as shown in Figure 6. Furthermore, the pump fluctuations, which are part of $\Delta u$, are removed effectively. Pump fluctuations result in uniform velocity changes in the axial direction whereas turbulent structures do not.

The series of measurements for unsteady flow is conducted over a range of mean Reynolds numbers $R e_{m}$ for a single velocity amplitude, i.e., $R e_{o}=610$ and four Womersley numbers $(\alpha=10-25)$ representative for blood flow within the aorta and arteries. Measurements are conducted phase-locked at eight instances during each flow cycle. The required measurement frequency and total measurement time for each case are shown in Table I.

Based on the modified velocity fluctuation field $\psi_{\text {mod }}^{\prime}$, the turbulence intensity is calculated at the centerline. The turbulence intensity is normalized by the centerline velocity and averaged over time. The results are shown in Figure 7. The results for the steady case, i.e., the result described in Sec. IV B, are shown as open circles $(\alpha=0)$. Compared to the results based on the radial velocity fluctuation component, discussed in Sec. IV B, the turbulence intensity at the centerline for steady laminar flow has decreased to a value of $I \approx 0.1 \%$. This observation confirms that the new method to compute the velocity fluctuation does indeed also provide a correction for pump fluctuations. 
TABLE I. Measurements of unsteady pulsatile flow with a Womersley number $\alpha$, where $f$ is the frequency of the pulsation, $f_{t}$ the frequency of measurements, $T_{t}$ the total measurement time based on 50 image pairs per phase, and $u^{\prime} / D$ an estimate of the reciprocal value of the turbulence integral time-scale.

\begin{tabular}{lcccc}
\hline \hline$\alpha$ & $f$ & $f_{t}$ & $T_{t}$ & $u^{\prime} / D$ \\
{$[-]$} & {$[1 / \mathrm{s}]$} & {$[1 / \mathrm{s}]$} & {$[\mathrm{s}]$} & 0.0775 \\
\hline 0 & 0.0000 & 1.000 & 50 & 0.0900 \\
10 & 0.0398 & 0.318 & 1256 & 0.0692 \\
15 & 0.0895 & 0.716 & 559 & 0.0825 \\
20 & 1.273 & 314 & 0.0955 \\
25 & 0.1592 & 1.989 & 201 & \\
\hline \hline
\end{tabular}

In order to investigate the transitional flow, the turbulence intensity $I$ as function of time for several mean Reynolds numbers within the transitional range are shown in Figure 8. For $R e_{m}=2232$, a single spike in the turbulence intensity can be observed at $t / T \approx 8$. The turbulence intensity is close to zero for the remainder of the observation, indicating laminar flow. For increasing $R e_{m}$, the number of spikes increases. For $R e_{m}=2527$, the increased turbulence intensity persists for a longer period of time. For $R e_{m}=2761$, the turbulence intensity of the flow remains $I \approx 3 \%$ for the duration of the observation, indicating fully developed turbulent flow.

The spikes of turbulence intensity for $R e_{m}<2761$ occur at relatively low Reynolds numbers and are caused by the inlet conditions (i.e., the orifice plate). The localized nature is indicative for the presence of a turbulent puff. The turbulence intensity within a puff is expected to be several times larger than the turbulence intensity for a fully turbulent flow, ${ }^{17}$ something clearly shown by the spikes of turbulence intensity in Figure 8.

Due to the difference in measurement frequency, see Table I for an overview, a puff is captured in more instantaneous velocity fields for higher Womersley numbers. For the unsteady cases, the total measurement time is long compared to the survival time of a puff. ${ }^{35}$ As a result, the statistics average out with little influence of individual puffs. For the steady case, however, the total measurement time is short compared to the survival time. This means that a puff (if present) will be present in a larger fraction of the total measurement time. This means that the statistics will be more strongly influenced by the possible presence of a puff. As a result, the transition (as reported in Figure 7) seems to be less gradual. This hypothesis is underpinned by evaluating the data that is associated with the apparent outlier marked with an arrow in Figure 7, for $\left(R e_{m}=2237\right.$ and $\left.\alpha=0\right)$. Visual inspection of the velocity fields supports the conclusion that this "outlier" is the result of a

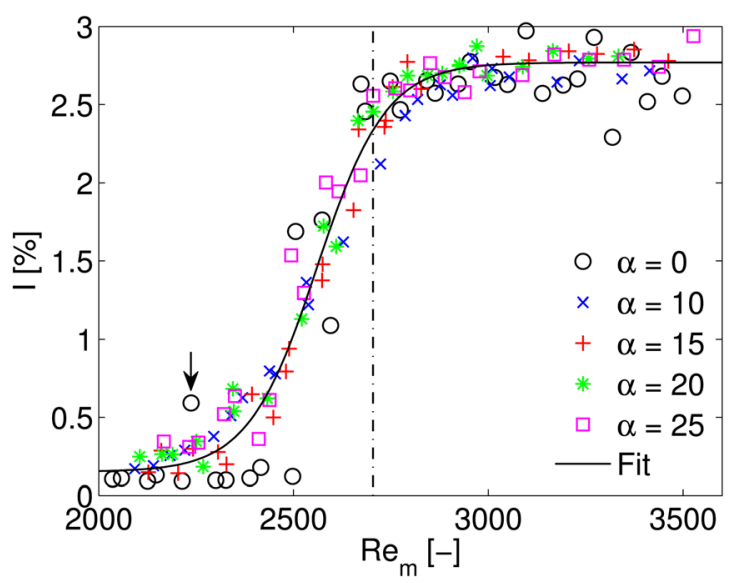

FIG. 7. (Color online) Turbulence intensity $I$ at the centerline based on $\psi_{\bmod }^{\prime}$ normalized by the centerline velocity as function of the mean Reynolds number $R e_{m}$ for five different Womersley numbers. The dotted line indicates the mean Reynolds number for which the dependence on oscillatory Reynolds number is shown in Figure 10. The arrow marks a specific measurement discussed in the text. 


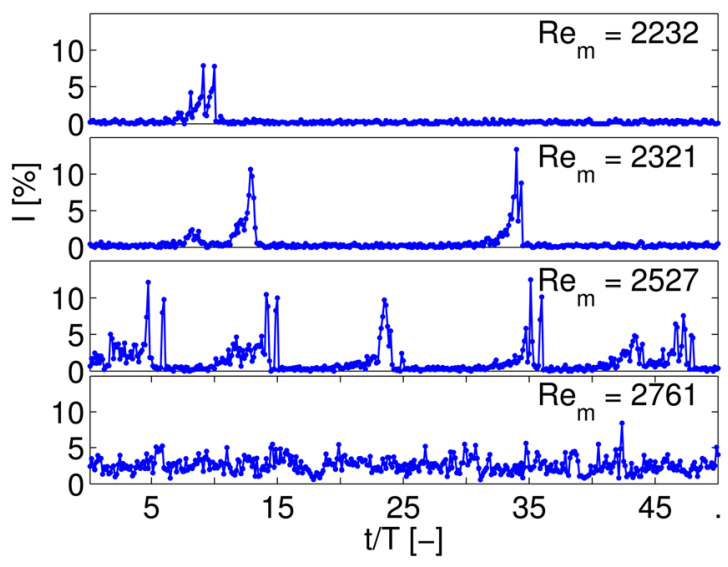

FIG. 8. (Color online) Turbulence intensity $I$ as function of time $t / T$ for four different mean Reynolds numbers $R e_{m}$, oscillatory Reynolds number $R e_{o}=610$ and $\alpha=25$.

single puff. This problem can be overcome by recording significantly more image pairs, but this turned out to be unfeasible in practice.

To confirm the presence of puffs, the flow is visualized using Iriodin flakes. For both steady and unsteady transitional flow, a series of images is recorded with a frame rate of $f_{t}=10 \mathrm{~Hz}$ to capture the passage of a puff. To obtain a spatial reconstruction, images are stitched together with a spatial distance based on the average velocity of the puff which is approximately equal to the bulk velocity. In Figure 9, the characteristic shape of a puff ${ }^{36}$ is clearly recognizable for both steady as well as unsteady flow, i.e., a conical tail of turbulence at the centerline downstream side of the puff and initial turbulence at the wall at the upstream side. The figure also shows velocity fluctuations in the radial directions measured with PIV. The PIV data are combined in a similar way to the visualization images. The velocity data appears to show a much smaller puff, but this is actually caused by the large velocity fluctuations in the core of the puff compared to the fluctuations in its downstream tail. Visual inspection of the data showed that the flow consisted of small, isolated turbulent structures; we did not observe growing slug-like structures that start to appear in steady flows at Reynolds numbers above $2400-2700 .^{2,17}$ A detailed comparison of the full flow structure of the puffs in the steady and pulsatile cases would be interesting, but cannot be done with the present measurement facilities. A detailed study using e.g., high-speed stereoscopic PIV

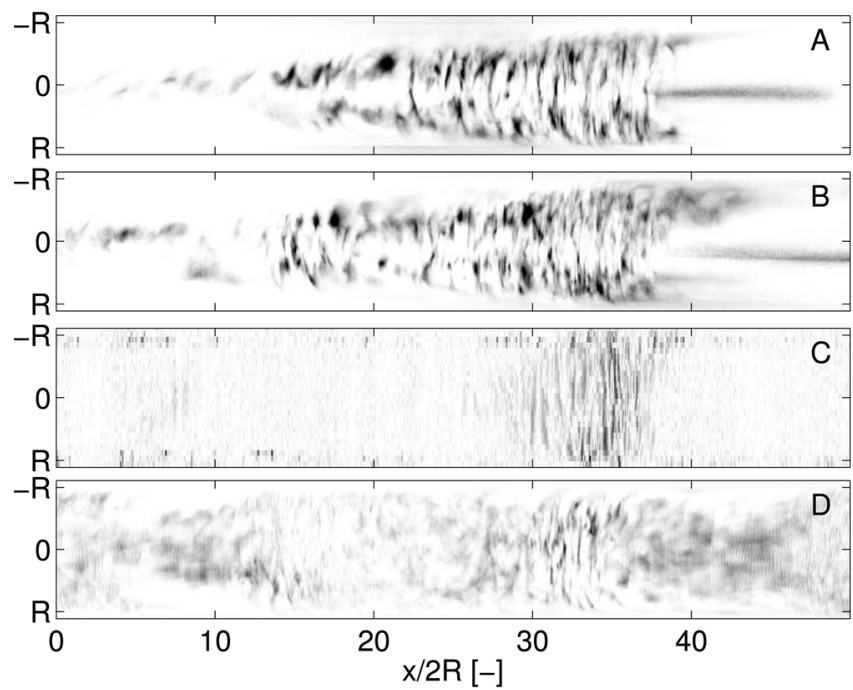

FIG. 9. A visualization of a puff for unsteady (A) and steady (B) base flow. Image (C) gives the velocity fluctuations in the radial direction obtained by PIV. The phase-locked turbulence regime is shown in (D). The flow direction is from right to left. (Notice that the horizontal axis is compressed relative to the vertical axis). 


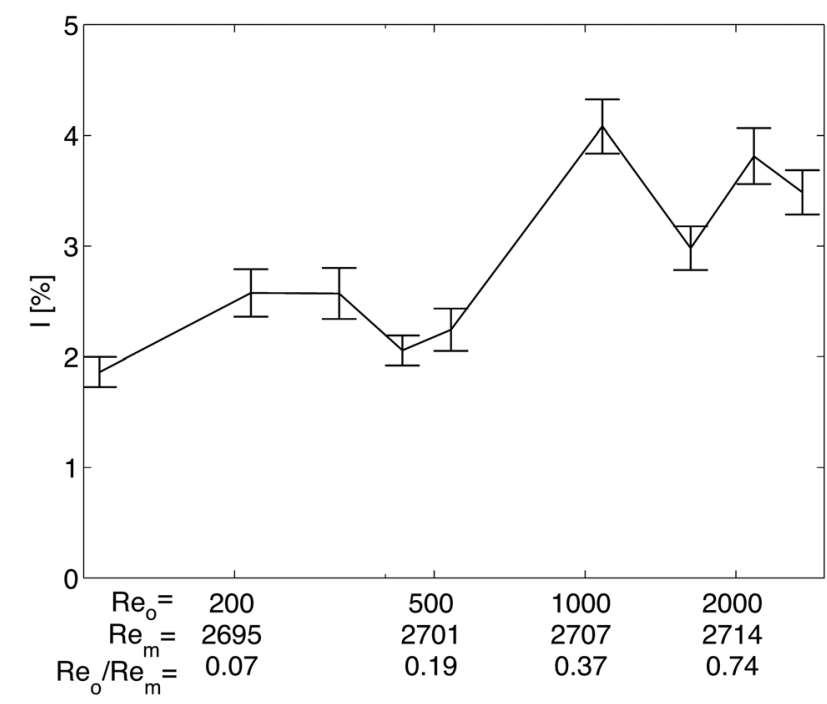

FIG. 10. Turbulence intensity $I$ as a function of the oscillating Reynolds number $R e_{o}$. The corresponding temperaturecorrected mean Reynolds numbers $R e_{m}$ and the ratio $R e_{o} / R e_{m}$ are also indicated.

(Ref. 36) would only allow measurement of a very limited number of puffs, which inhibits a study of the statistics as we do here.

Returning to Figure 7, no clear differences can be observed for the different pulsatile flows. Therefore, a single fit through all values is shown in Figure 7. This is in agreement with results of Stettler and Hussain ${ }^{15}$ who found that the influence of pulsation is limited to $\alpha<10$. The s-like shape of the turbulence intensity graphs (Figures 5 and 7) resembles the graph of the intermittency as a function of Reynolds number as reported in Yellin, ${ }^{18}$ data which was obtained by flow visualization. This confirms our hypothesis that the increase in puff number causes the smooth increase in turbulent intensity.

Based on the turbulence integral time-scale shown in Table I, which is of the same order of magnitude as the time scale of the pulsation, one would expect an influence for $\alpha<10$. Peacock et al. ${ }^{16}$ mention that the limited effect is caused by the use of a grid or orifice plate, which causes a lower critical Reynolds number due to the presence of long living transients. ${ }^{37}$

The question arises if the oscillatory Reynolds number influences the turbulence intensity for a fixed mean Reynolds number. For $R e_{m} \approx 2700$, the turbulence intensity is shown in Figure 10 as a function of the oscillatory Reynolds number. At first sight, it appears that the turbulence intensity increases with $R e_{o}$. However, as the temperature was not completely constant during the measurement, the mean Reynolds number $R e_{m}$ increases slightly. Therefore, the observed increase in turbulence intensity is likely explained by the increase in the mean Reynolds number, rather than due to the different oscillatory Reynolds numbers (see e.g., the slope shown in Figure 7 for $R e_{m}=2700$, indicated by the dashed line). We, therefore, conclude that the turbulence intensity is not a function of the oscillating Reynolds number; this indirectly suggests that the transition behavior is also not influenced by this parameter. Figure 10 also shows that there is no clear relation between $R e_{o} / R e_{m}$ and the turbulence intensity.

\section{PHASE-LOCKED TURBULENCE}

For a Womersley number up to $\alpha=25$, phase-locked turbulence is expected for mean Reynolds number $R e_{m}>2700$ and oscillatory Reynolds numbers $R e_{o}>1400 .{ }^{15}$ None of the parameters $R e_{m}, R e_{o}, R e_{o} / R e_{m}$, or $\alpha$ gives a clear boundary between the two regimes indicated here. The phase-locked turbulence is a completely different phenomenon compared to the random occurrence of puffs. It is not likely that a local patch of turbulence that travels through the pipe appears phase-locked at a fixed measurement location for every flow cycle. A more likely explanation would be that the entire flow bursts into turbulence and then (partially) relaminarizes.

Two measurements are carried out within the phase-locked regime, for which the parameters are given in Table II. The PIV measurements shown are an average over 100 cycles and a $50 \%$ 
TABLE II. Measurements of unsteady pulsatile flow with Womersley number $\alpha$, the frequency of the pulsation $f$, the frequency of measurements $f_{t}$ based on the number of phase-locked measurements per cycle, and $u^{\prime} / D$ an estimate of the reciprocal value of the turbulence integral time-scale.

\begin{tabular}{lcccccr}
\hline \hline$R e_{m}$ & $R e_{o}$ & $R e_{o} / R e_{m}$ & $\alpha$ & $f$ & $f_{t}$ & $u^{\prime} / D$ \\
{$[-]$} & {$[-]$} & {$[-]$} & {$[-]$} & {$[1 / \mathrm{s}]$} & 0.1000 \\
\hline 4000 & 1610 & 0.40 & 15 & 0.0895 & 3.580 & 0.1350 \\
5000 & 4000 & 0.80 & 20 & 0.1592 & 3.184 & {$[1 / \mathrm{s}]$} \\
\hline \hline
\end{tabular}

overlap of the interrogation areas is used to obtain a higher resolution in the radial direction, required to resolve the radial dependence of the turbulence intensity. The bulk velocity is used as the reference velocity to define the turbulence intensity. Besides the PIV measurements, a flow visualization is carried out as well.

To analyze the occurrence of turbulence as a function of the phase of pulsatile flow, it is worthwhile to look at the turbulence characteristics over the pipe radius as function of phase first, as shown in Figures 11 and 12.

For a number of phases in both cases a small "bump" in $\left\langle u^{2}\right\rangle^{\frac{1}{2}}$ and $\left\langle v^{2}\right\rangle^{\frac{1}{2}}$ is shown around $r=3 / 4 R$. These are actually artifacts caused by reflection of light in the raw PIV images that are insufficiently corrected for by image background reduction. A reflection causes the mean velocity to decrease as reflections are stationary. When the mean velocity is subtracted this leads to a higher apparent fluctuating component.

The magnitude of the turbulence fluctuations near the center is comparable. Near the wall, $\left\langle u^{\prime 2}\right\rangle^{\frac{1}{2}}$ starts to increase at maximum acceleration. This increase continues until maximum deceleration of the flow is reached. This is more clearly observed for $R e_{m}=5000$. As soon as $\left\langle u^{\prime 2}\right\rangle^{\frac{1}{2}}$ starts to increase near the wall, the turbulence intensity increases towards the center of the pipe as well throughout the cycle. This is consistent with the concept of transport. This is also observed for the radial component, which increases as the axial velocity fluctuations increase towards the center.

To characterize the turbulent intensity in the entire pipe, the turbulence intensity is integrated over the pipe radius. Because the turbulence intensity in the azimuthal direction cannot be measured with planar PIV, it was assumed to be equal to the radial turbulence intensity because the mean velocity is zero in this direction as well. The turbulence intensity during a cycle for both

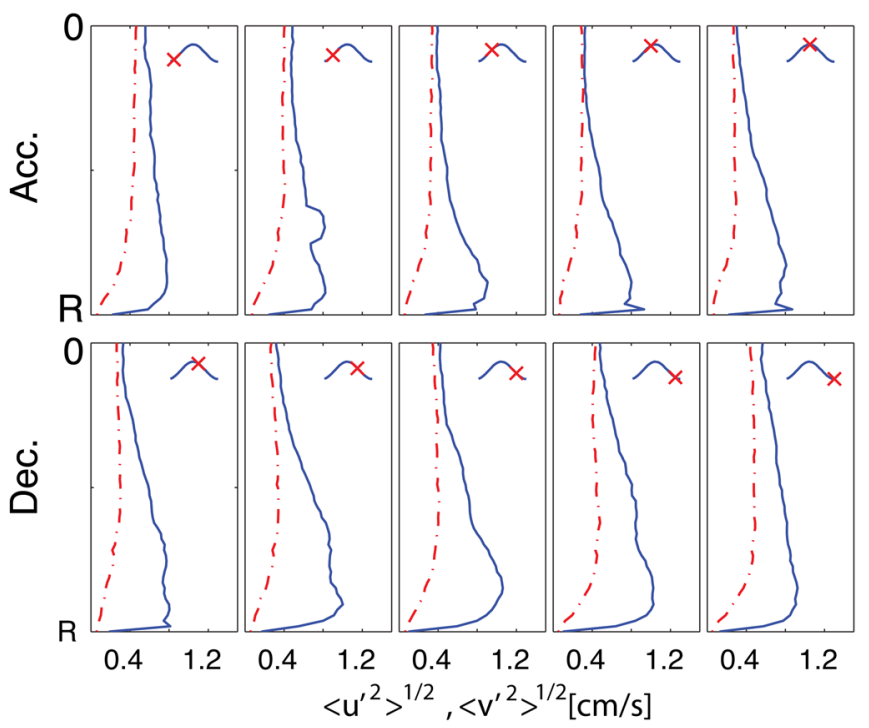

FIG. 11. (Color online) $\left\langle u^{\prime 2}\right\rangle^{\frac{1}{2}}$ and $\left\langle v^{2}\right\rangle^{\frac{1}{2}}$ as function of time over the radial position $r$ with $R$ the pipe radius for $R e_{m}=4000, R e_{o}=1610$, and $\alpha=15$. The accelerating (Acc.) and decelerating phases (Dec.) are shown at the top and bottom rows, respectively, with an indication of the phase with respect to the bulk velocity flow cycle in the insets of each sub-image. 


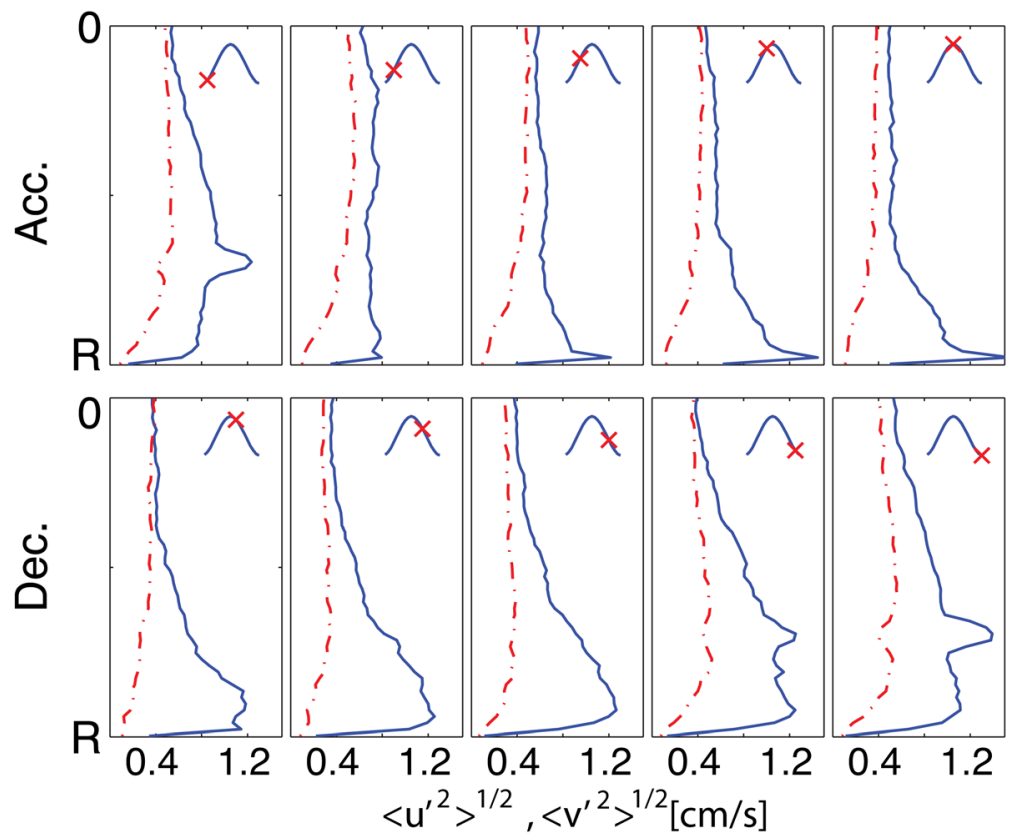

FIG. 12. (Color online) $\left\langle u^{2}\right\rangle^{\frac{1}{2}}$ and $\left\langle v^{\prime 2}\right\rangle^{\frac{1}{2}}$ as function of time over the radial position $r$ with $R$ the pipe radius for $R e_{m}=5000, R e_{o}=4000$, and $\alpha=20$. The accelerating (Acc.) and decelerating phases (Dec.) are shown at the top and bottom rows, respectively, with an indication of the phase with respect to the bulk velocity flow cycle in the insets of each sub-image.

$R e_{m}=4000$ and $R e_{m}=5000$ are shown in Figures 13 and 14 . The turbulence intensity has here been determined by averaging the RMS value for all realizations within one phase.

The turbulence intensity decreases during the accelerating phase and starts to increase during deceleration. A maximum is reached just before the end of the deceleration phase after which it remains constant. For $R e_{m}=5000$ a decrease is shown for the remainder of the cycle. Both cases show that the turbulence intensity maintains the same phase lag relative to the velocity. This observation is in excellent agreement with results shown by Ramaprian and $\mathrm{Tu},{ }^{20}$ but one should notice that their results concerned the centerline fluctuations only. Akhavan et al. ${ }^{38}$ report the turbulent kinetic energy integrated over the cross section, but these results are opposite to the results shown here. Maximum turbulent kinetic energy was found at the end of the accelerating phase instead of at the moment of largest deceleration. A possible explanation is the oscillatory nature of

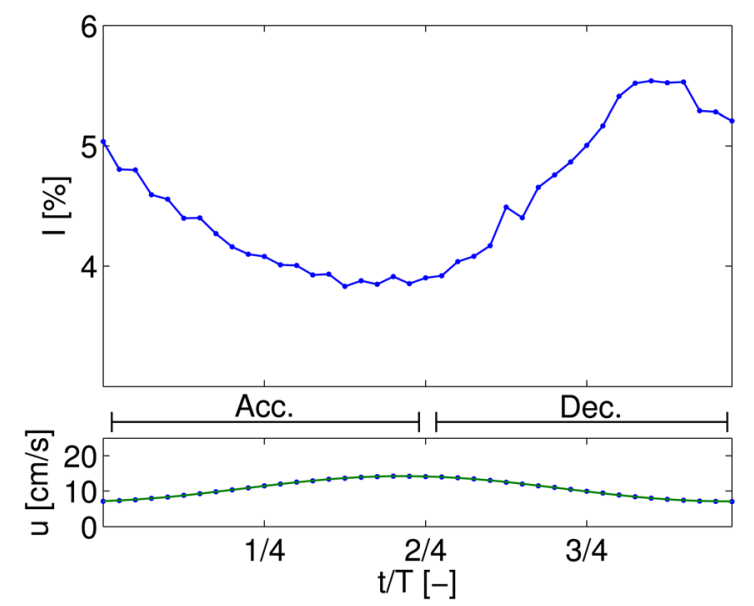

FIG. 13. (Color online) Turbulence intensity $I$ as function of time $t / T$ for $R e_{m}=4000, R e_{o}=1610$, and $\alpha=15$. The bottom figure shows the bulk velocity to indicate the flow cycle. 


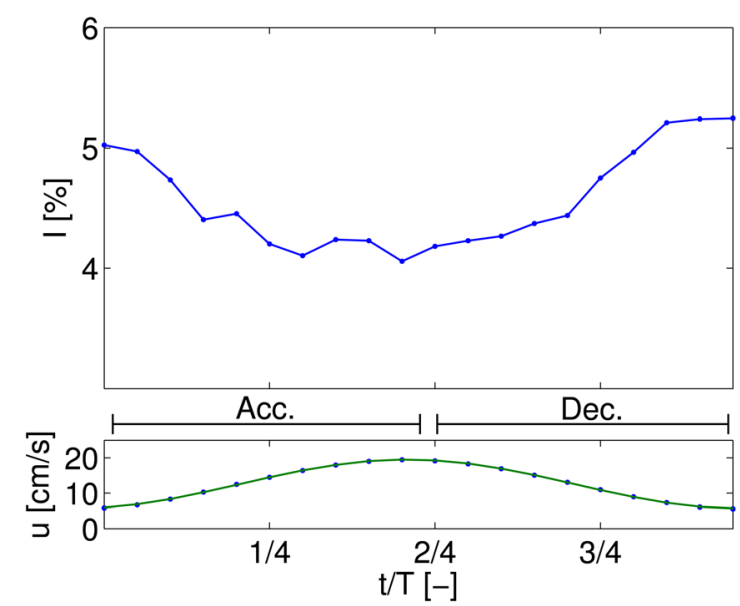

FIG. 14. (Color online) Turbulence intensity $I$ as function of time $t / T$ for $R e_{m}=5000, R e_{o}=4000$, and $\alpha=20$. The bottom figure shows the bulk velocity to indicate the flow cycle.

their flow, i.e., no mean velocity component, instead of the pulsatile flow studied here. In the present case, even during the decelerating phase, the velocity always has the same sign.

A plausible explanation for the relation between velocity and turbulence intensity is given by Bluestein and Einav. ${ }^{39}$ It is mentioned that disturbances are generally observed to occur during flow deceleration. During deceleration, the flow exhibits inflection points in the velocity profiles, which is a condition that might lead to an unstable flow. As the velocity approaches a minimum, the free stream can no longer feed the turbulence and the turbulent structures that have just formed start to decay. Whether the flow will completely relaminarize during the accelerating phase probably depends on the oscillating frequency, since it takes some time for turbulence to decay. For the cases examined here, the flow does not completely relaminarize. This can be explained by the turbulence integral time-scale, which is of the same order of magnitude as the frequency of the pulsation (see Table II). Simply stated, there is simply not enough time for turbulence to decay completely.

Whether turbulent patches are present or the flow bursts into turbulence over, the entire pipe at once is difficult to conclude from the PIV measurements recorded for a relatively small observation window. Flow visualization using Iriodin flakes was again used to observe the transition over the entire pipe length at once. Visual inspection (see e.g., Figure 9(D)) show that the flow does not relaminarize completely, but remains turbulent. The structures during the accelerating phase are large compared to the pipe radius. It is not possible to conclude whether the structures are located near the wall. When the minimum velocity has been nearly reached the flow abruptly burst into small structures at apparent random axial locations within the entire pipe, swiftly growing throughout the entire pipe length.

\section{CONCLUSIONS}

The transition of a pulsatile flow has been investigated in two distinct regimes. Within the first regime, where transition from laminar to turbulent flow occurs, a laminar, transitional, and turbulent region could be distinguished. These regions are described only by the mean Reynolds number; neither the Womersley number nor the oscillatory Reynolds number has an influence in the range that is studied. For transitional mean Reynolds numbers, the PIV measurements show the presence of puffs, their number increasing with increasing mean Reynolds number. This behavior is similar to what has been observed in steady flow experiments. These puffs are evident in flow visualization experiments as well.

The lack of influence of the Womersley number suggests that the trend of decreasing critical Reynolds number with increasing pulsatile effects that was observed in classic studies ${ }^{12-14}$ does not continue in the range of larger values of $\alpha$ that are studied here. Stettler and Hussain ${ }^{15}$ found 
similar results, with little influence for values of $\alpha>10$. Interestingly, they also found a region $4<\alpha<8$ where transition is suppressed. This particular regime will be part of future studies.

For the second regime, the turbulence intensity is phase-locked with the flow cycle. It has a phase lag with respect to the mean velocity, which appears to be independent of the mean Reynolds number, oscillatory Reynolds number, and Womersley number. Flow visualization shows a burst into turbulence at random axial positions swiftly growing to fill the entire pipe. The turbulence intensity decreases during the accelerating part of the cycle and increases in the first part of the decelerating part. A more detailed, quantitative description might be provided by analyzing the energy production and dissipation during a cycle. This requires knowledge of the full velocity gradient tensor. The latter can be provided by e.g., multi-plane stereo-PIV or tomographic PIV measurements, which are planned for future work. These experiments will provide all information required to measure the exact energy budgets.

${ }^{1}$ P. D. Stein and H. N. Sabbah, "Turbulent blood flow in the ascending aorta of humans with normal and diseased aortic valves," Circulation Res. 39(1), 58 (1976).

${ }^{2}$ K. Avila, D. Moxey, A. de Lozar, M. Avila, D. Barkley, and B. Hof, "The onset of turbulence in pipe flow," Science 333(6039), 192 (2011).

${ }^{3}$ B. Eckhardt, T. M. Schneider, B. Hof, and J. Westerweel, "Turbulence transition in pipe flow," Annu. Rev. Fluid Mech. 39, 447 (2007).

${ }^{4}$ T. Mullin, "Experimental studies of transition to turbulence in a pipe," Annu. Rev. Fluid Mech. 43, 1 (2011).

${ }^{5}$ M. Ö. Çarpinlioğlu and M. Y. Gündoğdu, "A critical review on pulsatile pipe flow studies directing towards future research topics," Flow Meas. Instrum. 12(3), 163 (2001).

${ }^{6} \mathrm{~S}$. Uchida, "The pulsating viscous flow superposed on the steady laminar motion of incompressible fluid in a circular pipe,” Z. Angew. Math. Phys. 7(5), 403 (1956).

${ }^{7} \mathrm{~J}$. R. Womersley, "Method for the calculation of velocity, rate of flow and viscous drag in arteries when the pressure gradient is known," J. Physiol. 127(3), 553 (1955).

${ }^{8}$ M. Hino, M. Sawamoto, and S. Takasu, "Experiments on transition to turbulence in an oscillatory pipe flow," J. Fluid Mech. 75(2), 193 (1976).

${ }^{9}$ B. Ü. L. Ünsal and F. Durst, "Pulsating flows: Experimental equipment and its application," JSME Int. J., Ser. B 49(4), 980 (2006).

${ }^{10} \mathrm{~S}$. He and J. D. Jackson, “An experimental study of pulsating turbulent flow in a pipe,” Eur. J. Mech. B/Fluids, 28(2), 309 (2009).

${ }^{11}$ M. Nabavi and K. Siddiqui, "A critical review on advanced velocity measurement techniques in pulsating flows," Measurement Sci. Technol. 21, 042002 (2010).

${ }^{12}$ D. Hershey and C. S. Im, "Critical reynolds number for sinusoidal flow of water in rigid tubes," AIChE J. 14(5), 807 (1968).

${ }^{13}$ R. M. Nerem and W. A. Seed, "An in vivo study of aortic flow disturbances," Cardiovascular Res. 6(1), 1 (1972).

${ }^{14}$ H. L. Falsetti, R. J. Carroll, R. D. Swope, and C. J. Chen, "Turbulent blood flow in the ascending aorta of dogs," Cardiovasc. Res. 17(7), 427 (1983).

${ }^{15}$ J. C. Stettler and A. K. M. Hussain, “On transition of the pulsatile pipe flow,” J. Fluid Mech. 170(1), 169 (1986).

${ }^{16}$ J. Peacock, T. Jones, C. Tock, and R. Lutz, "The onset of turbulence in physiological pulsatile flow in a straight tube," Exp. Fluids 24(1), 1 (1998).

${ }^{17}$ I. J. Wygnanski and F. H. Champagne, "On transition in a pipe. Part 1. The origin of puffs and slugs and the flow in a turbulent slug,” J. Fluid Mech. 59(2), 281 (1973).

${ }^{18}$ E. L. Yellin, "Laminar-turbulent transition process in pulsatile flow," Circulation Res. 19(4), 791 (1966).

${ }^{19}$ M. Clamen and P. Minton, "An experimental investigation of flow in an oscillating pipe," J. Fluid Mech. 81(03), 421 (1977).

${ }^{20}$ B. R. Ramaprian and S. W. Tu, "Fully developed periodic turbulent pipe flow. Part 2. The detailed structure of the flow," J. Fluid Mech. 137, 59 (1983).

${ }^{21}$ M. Iguchi, G. Park, and Y. Koh, "The structure of turbulence in pulsatile pipe flows," J. Mech. Sci. Technol. 7(3), 185 (1993).

${ }^{22}$ D. M. Eckmann and J. B. Grotberg, "Experiments on transition to turbulence in oscillatory pipe flow," J. Fluid Mech. 222(1), 329 (1991).

${ }^{23}$ F. Fedele, D. L. Hitt, and R. D. Prabhu, "Revisiting the stability of pulsatile pipe flow," Eur. J. Mech. B/Fluids, 24(2), 237 (2005).

${ }^{24}$ W. H. Yang and C. S. Yih, "Stability of time-periodic flows in a circular pipe," J. Fluid Mech. 82, 497 (1977).

${ }^{25}$ T. S. Lee and Z. D. Shi, "Numerical study of effects of pulsatile amplitude for transitional turbulent pulsatile flow in pipes with ring-type constrictions," Int. J. Numer. Methods Fluids 30(7), 813 (1999).

${ }^{26}$ Y. C. Fung, Biodynamics Circulation (Springer-Verlag, New York, 1984).

${ }^{27}$ A. Hussain and W. C. Reynolds, "The mechanics of an organized wave in turbulent shear flow," J. Fluid Mech. 41(2), 241 (1970).

${ }^{28}$ A. A. Draad, G. D. C. Kuiken, and F. T. M. Nieuwstadt, "Laminar-turbulent transition in pipe flow for Newtonian and non-Newtonian fluids," J. Fluid Mech. 377(1), 267 (1998).

${ }^{29}$ F. Durst and B. Ü. L. Ünsal, "Forced laminar-to-turbulent transition of pipe flows," J. Fluid Mech. 560, 449 (2006).

${ }^{30}$ R. J. Adrian and J. Westerweel, Particle Image Velocimetry (Cambridge University Press, United Kingdom, 2010).

${ }^{31}$ J. Westerweel, D. Dabiri, and M. Gharib, "The effect of a discrete window offset on the accuracy of cross-correlation analysis of digital PIV recordings," Exp. Fluids, 23(1), 20 (1997).

${ }^{32}$ J. Westerweel and F. Scarano, "Universal outlier detection for PIV data," Exp. Fluids 39(6), 1096 (2005). 
${ }^{33}$ C. Poelma, J. Westerweel, and G. Ooms, "Turbulence statistics from optical whole-field measurements in particle-laden turbulence," Exp. Fluids 40(3), 347 (2006).

${ }^{34}$ S. Pope, Turbulent Flows (Cambridge University Press, Cambridge, UK, 2000).

${ }^{35}$ D. J. Kuik, C. Poelma, and J. Westerweel, "Quantitative measurement of the lifetime of localized turbulence in pipe flow," J. Fluid Mech. 645(1), 529 (2010).

${ }^{36}$ C. W. H. van Doorne and J. Westerweel, "The flow structure of a puff,". Philos. Trans. R. Soc. A 367(1888), 489 (2009).

${ }^{37}$ A. M. Binnie and J. S. Fowler, "A study by a double-refraction method of the development of turbulence in a long circular tube," Proc. R. Soc. London, Ser. A 192(1028), 32 (1947).

${ }^{38}$ R. Akhavan, R. D. Kamm, and A. H. Shapiro, "An investigation of transition to turbulence in bounded oscillatory Stokes flows Part 1. Experiments," J. Fluid Mech. 225, 395 (1991).

${ }^{39}$ D. Bluestein and S. Einav, "Techniques in the analysis of stability of pulsatile flow through heart valves", in Biomechanical Systems: Techniques and Applications: Cardiovascular Techniques, Vol. II, edited by C. T. Leondes (CRC Press LLC, Boca Raton, FL, 2001), pp. 4-1-4:39. 